\title{
Cytokines in pulmonary arterial hypertension: consider sensitivity when using multiplex technology
}

\begin{abstract}
To the Editor:
CRACOWSKI et al. [1] have recently published an interesting substudy in which they analysed the association of 17 baseline plasma cytokine concentrations with mortality in patients suffering from pulmonary arterial hypertension $(\mathrm{PAH})$, using multiplex technology. The authors showed that four cytokines were independently associated with an increase in the adjusted hazard of mortality. Previously, Soon et al. [2] demonstrated that elevated levels of cytokines in PAH also predict survival over a 5-year period. Both these studies reinforce the increasing evidence that inflammation is associated with PAH remodelling.
\end{abstract}

Nevertheless, it is known that concentrations of circulating cytokines are very low, in the $\mathrm{pg} \cdot \mathrm{mL}^{-1}$ range, requiring highly sensitive methods. In the letter by CRACOWSKI et al. [1], despite a well-defined design and a robust statistical analysis, as previously described [3], no information is provided about the analytical performances of the method for cytokine quantification. In order to strengthen such cytokine-based clinical studies, the sensitivity of multiplex technology is herein discussed.

Table 1 shows the mean baseline circulating cytokine concentrations from the two predictive PAH-related studies of CracowsKi et al. [1] and SoOn et al. [2], using different multiplex technologies: the BiochipsArray from Randox (Crumlin, UK) and the plate-based Multi-Array from Meso Scale Discovery (MSD; Rockville, MD, USA), respectively. In addition, the table 1 details, for each cytokine, the sensitivities reported by the manufacturers, and those determined in analytical studies by FITZGERALD et al. [4] and DABITAO et al. [5].

The theoretical sensitivity, also known as the lower limit of detection (LLOD), is defined as the lowest concentration of the analyte that can be distinguished from zero with at least two standard deviations, whereas the functional sensitivity, also known as the lower limit of quantification (LLOQ), is defined as the lowest concentration giving a coefficient of variation $<20 \%$ on replicates. In hospital practice, for a given parameter measured in biological fluids, a result below the sensitivity of the method should neither be validated nor transmitted to the clinicians, because of its excessive imprecision. As shown in table 1, among the four cytokines independently associated with death in the study by CRACOWSKI et al. [1], three had a mean baseline concentration below the corresponding sensitivities claimed by Randox (interleukin (IL)-1 $\alpha$, IL-13 and tumour necrosis factor- $\alpha$ ) and two of these are also below the functional sensitivities as determined by FitzGerald et al. [4]. Using the same Randox system, DunCAN et al. [6] have shown that IL-6 and vascular endothelial growth factor were significantly associated with the occurrence of an adverse event in paediatric $\mathrm{PAH}$, with median concentrations very closed to the sensitivities claimed by the manufacturer. In contrast, considering the PAH patients from the study by SoON et al. [2], the concentrations of the four cytokines independently linked with survival time are much higher than the LLOQs reported by MSD or than those determined by DABITAO et al. [5], making these results absolutely valid, analytically. It should be noted that most of the standard deviations are very high, suggesting a skewed distribution, with a few patients with very high levels and a non-negligible number of patients with cytokine concentrations below the limit of detection $[1,2]$. This high number of nondetected data $(>50 \%)$ has been recently described for cytokines assessed by three commercially available multiplex assays [7]. Consequently, caution should be considered about low cytokine circulating concentrations, because most probably measured with an analytical imprecision $>20 \%$, making such promising clinical findings somewhat questionable.

Functional sensitivity should be considered in clinical studies, especially when the main outcome is based on cytokine measurement. This criterion could be determined by repeated measurements of plasma pools, prepared so as to obtain decreasing concentrations of cytokine, allowing determination of the lowest concentration giving an imprecision of $20 \%$ using polynomial regression or Horwitz curve, as recommended by the French Accreditation Committee (COFRAC). Such a protocol has been recommended by the National Academy of Clinical Biochemistry for the determination of functional sensitivity of the third-generation thyroid-stimulating hormone test, as recently published [8]. Elsewhere, a similar approach has been used to determine the sensitivity of the high-sensitivity cardiac troponin T test [9]. 


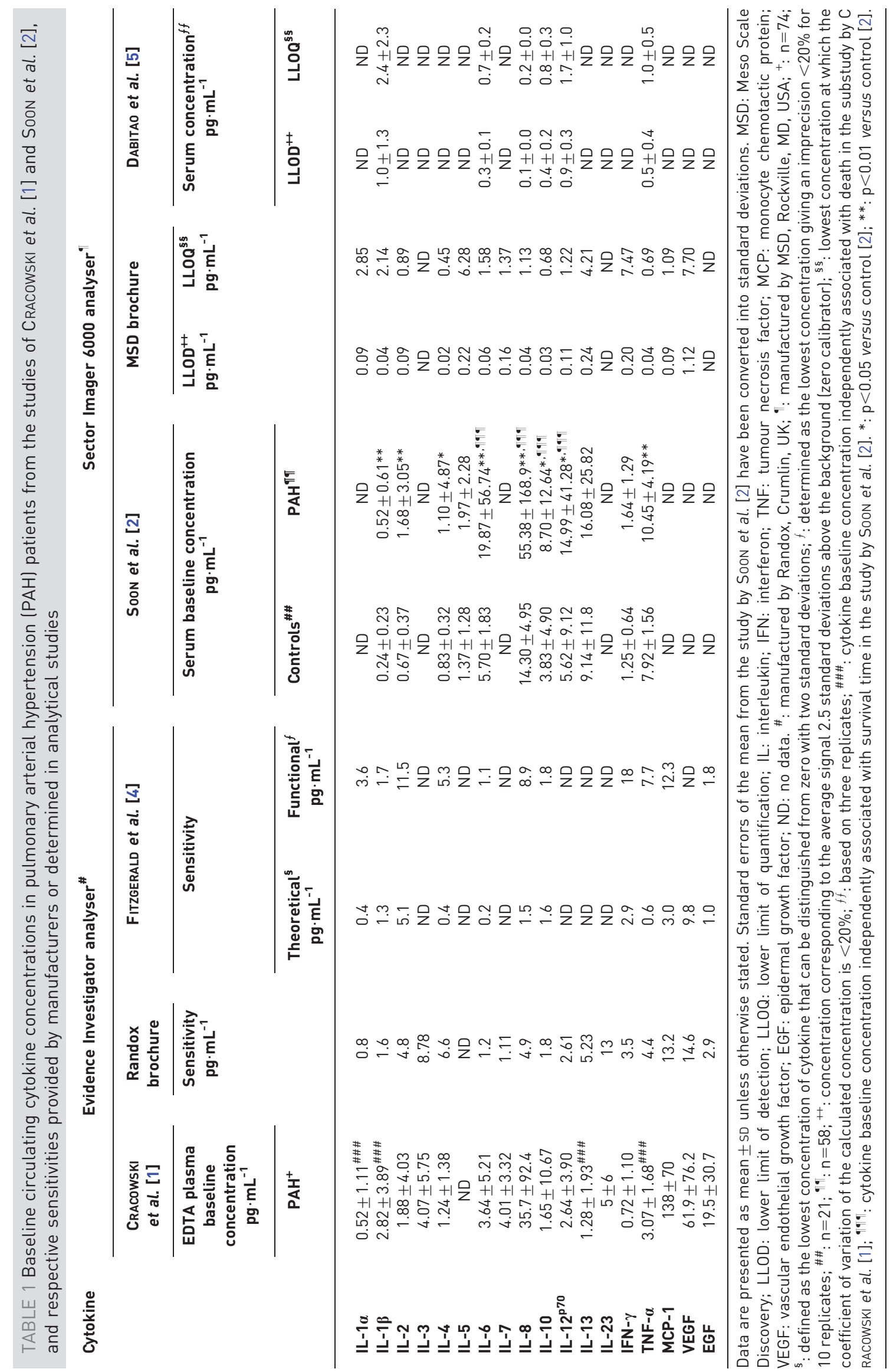


Recently, using the expensive and time-consuming ELISA method, HerEsI et al. [10] have shown that plasma IL- 6 concentrations $>4.7 \mathrm{pg} \cdot \mathrm{mL}^{-1}$ provide incremental prognostic information in $\mathrm{PAH}$, with valid concentrations above the theoretical sensitivity (LLOD $0.7 \mathrm{pg} \cdot \mathrm{mL}^{-1}$ ). Similarly, using MSD multiplex technology, Soon et al. [2] showed that serum IL-6 was also associated with survival in PAH patients, with concentrations $\left(19.87 \pm 7.45 \mathrm{pg} \cdot \mathrm{mL}^{-1}\right.$ ) above the sensitivity (LLOQ $1.58 \mathrm{pg} \cdot \mathrm{mL}^{-1}$ ), making such a cytokine very promising for the prognosis of $\mathrm{PAH}$.

In conclusion, multiplex analysis provides much information from a single biological sample, and is therefore popular and more frequently used in PAH studies. However, cytokine concentrations are low and often close to the sensitivity of the assay, depending on the multiplex assays. This currently prevents the routine clinical use of such biomarkers, and should encourage clinicians to take advantage of the biochemists' analytic experience.

@ERSpublications

Sensitivity must be considered for cytokines when using multiplex technology in PAH-related clinical studies http://ow.ly/tAT91

Denis Monneret

Laboratory of Metabolic Biochemistry, La Pitié Salpêtrière-Charles Foix University Hospital (AP-HP), Paris, France.

Correspondence: Denis Monneret, La Pitié Salpêtrière-Charles Foix University Hospital (AP-HP), 47-83 Boulevard de l’Hôpital, Paris, 75013, France. E-mail: dmonneret2@gmail.com

Received: Jan 012014 | Accepted after revision: Feb 072014

Conflict of interest: None declared.

\section{References}

1 Cracowski JL, Chabot F, Labarere J, et al. Proinflammatory cytokine levels are linked with death in pulmonary arterial hypertension. Eur Respir J 2014; 43: 915-917.

2 Soon E, Holmes AM, Treacy CM, et al. Elevated levels of inflammatory cytokines predict survival in idiopathic and familial pulmonary arterial hypertension. Circulation 2010; 122: 920-927.

3 Cracowski JL, Degano B, Chabot F, et al. Independent association of urinary F2-isoprostanes with survival in pulmonary arterial hypertension. Chest 2012; 142: 869-876.

4 Fitzgerald SP, Lamont JV, McConnell RI, et al. Development of a high-throughput automated analyzer using biochip array technology. Clin Chem 2005; 51: 1165-1176.

5 Dabitao D, Margolick JB, Lopez J, et al. Multiplex measurement of proinflammatory cytokines in human serum: comparison of the Meso Scale Discovery electrochemiluminescence assay and the Cytometric Bead Array. J Immunol Methods 2011; 372: 71-77.

6 Duncan M, Wagner BD, Murray K, et al. Circulating cytokines and growth factors in pediatric pulmonary hypertension. Mediators Inflamm 2012; 143428.

7 Dupuy AM, Kuster N, Lizard G, et al. Performance evaluation of human cytokines profiles obtained by various multiplexed-based technologies underlines a need for standardization. Clin Chem Lab Med 2013; 51: 1385-1393.

8 Monneret D, Guergour D, Vergnaud S, et al. Evaluation of LOCI technology-based thyroid blood tests on the Dimension Vista analyzer. Clin Biochem 2013; 46: 1290-1297.

9 Giannitsis E, Kurz K, Hallermayer K, et al. Analytical validation of a high-sensitivity cardiac troponin T assay. Clin Chem 2010; 56: 254-261.

10 Heresi GA, Aytekin M, Hammel JP, et al. Plasma interleukin-6 adds prognostic information in pulmonary arterial hypertension. Eur Respir J 2014; 43: 912-914.

\section{Is auto-servoventilation unnecessary in patients with heart failure and apnoea?}

\section{To the Editor:}

We read with great interest the recent report by ARZT et al. [1]. They investigated the potential benefit of auto-servoventilation (ASV) in addition to an optimal medical management (OMM) on cardiac function and quality of life in patients with congestive heart failure (CHF) coexisting with central and obstructive sleep apnoea (COSA). As the interest of ASV remains debated in patients with CHF and "pure" central sleep apnoea [2], there is no clear evidence of the superiority of ASV over constant positive airway pressure (CPAP) in patients with CHF and COSA [3-5]. In our opinion, some aspects of the report by ARZT et al. [1] 